\title{
Effective segmentation of green vegetation for resource-constrained real-time applications
}

\author{
S. Moorthy ${ }^{1,3}$, B. Boigelot ${ }^{2}$ and B. C. N. Mercatoris ${ }^{3}$ \\ ${ }^{1}$ AgricultureIsLife Platform, Gembloux Agro-Bio Tech, Université de Liège, Belgium \\ ${ }^{2}$ Montefiore Institute, Université de Liège, Belgium \\ ${ }^{3}$ Biosystem Engineering Department, Gembloux Agro-Bio Tech, Université de Liège, \\ Belgium \\ sruthimoorthy.krishnamoorthyparvathi@ulg.ac.be
}

\begin{abstract}
This paper describes an improved algorithm for segmentation of green vegetation under uncontrolled illumination conditions and also suitable for resource-constrained real-time applications. The proposed algorithm uses a naïve Bayesian model to effectively combine various manually extracted features from two different color spaces namely RGB and HSV. The evaluation of 100 images indicated the better performance of the proposed algorithm than the vegetation index-based methods with comparable execution time. Moreover, the proposed algorithm performed better than the state-of-the-art EASA-based algorithms in terms of processing time and memory usage.
\end{abstract}

Keywords: Machine vision, Vegetation segmentation, Naïve Bayes, HSV color space.

\section{Introduction}

Recently, there has been increased interest in the development of small and low-cost robots performing various precision agriculture tasks in the field. Necessary information for the robot to navigate autonomously can be supplied by information-rich machine vision systems. An important issue is to develop a technique that recognizes the crop rows accurately and robustly with adequate tolerance to varying growth stages of the plants, poor and variable illumination conditions, missing crops and presence of weeds. Efficient and automatic segmentation of green vegetation from background is an important step not only for accurate recognition of crop rows, but also for many precision agriculture applications like weed detection for site-specific treatment. Despite recent developments, segmentation of green vegetation under uncontrolled illumination conditions in real time is still a major research challenge (Zheng et al, 2009).

Significant research has been undertaken on the segmentation of green vegetation for crop row line tracking and identification of single plants (crops and weeds) for applications such as precision spraying. Visible, spectral index-based methods, which have been proposed to segment green vegetation under variable illumination conditions include Excess Green $($ ExG) (Woebbecke et al, 1995), Color Index of Vegetation Extraction (CIVE) (Katoka et al, 2003), Excess Green minus Excess Red (ExGExR) (Camargo Neto, 2004) and Vegetative index (VEG) (Hague et al, 2006). A critical step is required to select the threshold value to binarize the near-binary image resulting from all the above spectral index-based methods. 
In addition to spectral index-based methods, the thresholding techniques proposed to segment crop images include dynamic thresholding method (Rovira-Mas et al, 2005), Otsubased thresholding methods (Meyer et al, 2008) and statistical mean-based segmentation of the image (Guijarro et al, 2011). These methods generally assume the histogram of the image to be bimodal and require the vegetation and background to belong to two different brightness regions. However, for outdoor images, the index intensities of the two regions frequently overlap owing to unstructured and non-uniform illumination conditions.

In recent years, research has been carried out on developing complex, yet efficient, algorithms for vegetation segmentation. These techniques include unsupervised fuzzy clustering algorithms intensified by Zadeh's intensification technique (Meyer et al, 2004), mean-shift-based learning procedure (Zheng et al, 2009) and Environmentally Adaptive Segmentation Algorithm (EASA) (Tian \& Slaughter, 1998).

Simple, threshold-based methods cannot completely separate the vegetation from the background under naturally changing outdoor lighting conditions. Therefore, the learningbased approaches prove to be a potential solution for vegetation segmentation under uncontrolled outdoor conditions. However, the state-of-the-art learning-based methods trade either memory or processing time for performance. The main objective of this study was to improve the segmentation of the green vegetation from soil by leveraging the information from different color spaces (RGB and HSV) for real-time applications without any compromise on the performance. The second objective of this study was to compare the performance of the proposed algorithm against the state-of-the-art visible spectral index-based methods for segmentation.

\section{Materials and methods}

Image acquisition

The images used for this study consisted of two types of vegetation namely sugar beet and maize. The images were captured with a low cost Fire-i ${ }^{\mathrm{TM}} 400$ industrial camera (manufactured by Unibrain S.A., Athens, Greece) with a $6 \mathrm{~mm}$ focal length lens. Images from sugar beet fields were captured between April to June 2014 in a 2.38-ha experimental field of Gembloux Agro-Bio Tech, Belgium. The images were acquired on seven different days accounting for the variability in the growth stage of the plants, illumination conditions and weed infestation rates. There was a gap of six to seven days between two consecutive acquisitions. Images from maize fields were captured during June 2014 in a commercial field in Gembloux when the maize plants were at their five-leaf stage. All the digital images were captured under perspective projection and stored as 24-bit RGB color images with a resolution of $640 \times 480$ in JPEG format ${ }^{1}$.

Image segmentation algorithm

The proposed algorithm consisted of two phases: a learning phase where a model was learned to detect the green vegetation and a segmentation phase where the learned model was used to segment the image into green and non-green parts.

1 The whole dataset collected from sugar beet and maize fields to do the experiments can be obtained by sending an email to the corresponding author. 
Learning phase. Figure 1 shows the two main steps involved in this phase: Extracting useful features and training the Bayesian classifier.

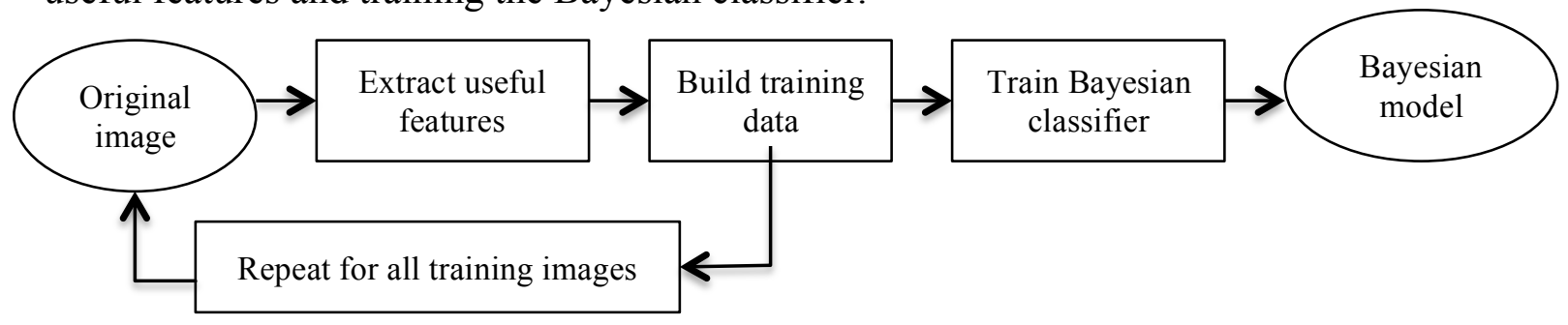

Figure 1. Steps involved in the learning phase of the image segmentation algorithm

As vegetation and background show great differences in the color, color information is considered to be useful and effective in segmentation. Despite giving color information, non-normalized RGB values are highly sensitive to the intensity of the illuminating source and illumination angles. Therefore, normalized RGB components were extracted as they eliminate the dependency on the illumination (Tian \& Slaughter, 1998). As HSV is a simple model of color with computational simplicity and is considered to be effective in object detection and segmentation, Hue (H), Saturation (S) and Value (V) components were extracted from the HSV color space.

In addition to the above-mentioned features, G-R (Green - Red) was also used as it gives the degree of greenness compared to the redness. Therefore, the final set of features used for segmentation consisted of: normalized (norm) RGB values, HSV components and G-R. A naïve Bayesian model was trained with these features to segment the images. Note that a naïve Bayesian model was also trained with the features norm RGB values and magnitude $\left(\sqrt{\left(R^{2}+G^{2}+B^{2}\right)}\right.$, where $\mathrm{R}, \mathrm{G}$ and $\mathrm{B}$ are not normalized). Since the results of the Bayesian model trained with norm RGB, HSV and G-R were superior to the one trained with norm RGB and magnitude, the latter was not finally considered.

A total of 500 images were used for training the classifier. For each image, an average of 3000 data points were randomly extracted with the aforementioned 7 features. Of the total $1,500,000$ data points used for training, $75 \%$ belonged to the background pixels accounting for the variability encountered in the background and the remaining $25 \%$ were from the green plants. To improve the segmentation rate of vegetation in the images, a naïve Bayesian algorithm was introduced in to the learning phase, as it has proved to work well in complex real-world problems (Hand \& Yu, 2001).

The naïve Bayes classifier estimates the a posteriori probability of a pixel belonging to one of the two classes (vegetation or background in this case) by using Bayes' rule assuming that the feature values are independent within a class.

As the feature values were continuous in this case, it is assumed that the feature values associated with each class follow a Gaussian distribution. Thus for each feature, mean and standard deviation associated with each class were computed from the training data and stored as shown in Table 1. The parameter estimation for each feature was based on the probability distribution of the feature (v) given the class $\mathrm{c}(\mathrm{c})$ as given in Eq.1. 


$$
p(V=v / c)=\frac{1}{\sqrt{2 \pi \sigma_{c}^{2}}} e^{\frac{-\left(v-\mu_{c}\right)^{2}}{2 \sigma_{c}^{2}}}
$$

where $\mu_{c}$ and $\sigma_{c}^{2}$ are the mean and variance of the feature $\mathrm{v}$ associated with the class $\mathrm{c}$.

Table 1. Mean and standard deviation of the features $\left(\mu_{c}, \sigma_{c}\right)$ used for segmenting the vegetation (Veg) from the background $(\mathrm{Bg})$

\begin{tabular}{llllllll}
\hline & norm R & norm G & Norm B & H & S & V & G-R \\
\hline Veg & $(0.32,0.03)$ & $(0.38,0.02)$ & $(0.29,0.04)$ & $(99.5,39)$ & $(26.3,11)$ & $(63.7,14)$ & $(23.4,11)$ \\
Bg & $(0.33,0.01)$ & $(0.32,0.01)$ & $(0.33,0.02)$ & $(5.9,44)$ & $(7.7,5)$ & $(69.3,15)$ & $(-6.7,10)$ \\
\hline
\end{tabular}

As can be seen from Table 1, the mean of the normalized green value was greater than the mean of the normalized red value for the vegetation class. In the background class, the normalized red value was dominant but only by a small quantity. It is worth mentioning about the hue, saturation and G-R values, as they were significantly greater for the vegetation class than for the background class. To further evaluate the merits of the features, a correlation-based feature selection (CFS) algorithm was applied to the dataset and the most useful set of features were found to be: hue, saturation, G and G-R (Hall \& Smith, 1998).

Segmentation phase. Once the Bayesian classifier had been trained to detect the green vegetation, the learned model was used for the decision making process. Given a new image, the main goal was to identify the pixels belonging to two classes namely vegetation and background. For each pixel in the new image, the seven features were extracted and the a posteriori probability of the two classes given the feature vector was estimated based on Eq. 2.

$$
p\left(c_{i} \mid v\right)=\frac{p\left(v \mid c_{i}\right) p\left(c_{i}\right)}{p(v)}
$$

where $\mathrm{v}$ is the feature vector containing norm RGB, HSV and G-R values, $\mathrm{c}$ is the class vector with the values vegetation and background and $p(v)=\sum_{i=1}^{N} p\left(v \mid c_{i}\right) p\left(c_{i}\right)$.

The most commonly used decision rule is to select the most probable hypothesis known as maximum a posteriori (MAP) decision rule and is given in Eq. 3.

$$
\text { Classify }(\mathrm{v})=\underset{c}{\operatorname{argmax}} P(C=c) \prod_{i=1}^{n} p\left(V_{i}=v_{i} Q_{=}\right)
$$

For each pixel in a new image, the features were extracted and the MAP decision rule given in Eq. 3 was applied to classify the pixel into vegetation or background.

Evaluation method

To evaluate the effectiveness of the proposed algorithm, the test images were manually segmented by hand-labeling each pixel with either 0 (background) or 255 (green vegetation). The image segmented by the Bayesian model was then compared to the handlabeled image. Accuracy as given in Eq. 4 was used as a statistical measure to evaluate the performance of the proposed algorithm. 
Accuracy $=\frac{\text { number of true positives }+ \text { number of true negatives }}{\text { Total number of pixels in the image }}$

The computer-segmented pixels were compared on a pixel-by-pixel basis to the handlabeled image to get the number of true positives and true negatives. An accuracy of 1.0 represents a perfect positive correlation between the segmentation by the proposed algorithm and manual segmentation, and vice versa.

\section{Results and discussion}

The proposed algorithm was implemented in a personal computer with an Intel core i7 processor at $2.6 \mathrm{GHz}$ and $8 \mathrm{~GB}$ RAM. The operating system was Mac OS X version 10.9.3. The proposed algorithm was implemented in Python with the open source computer vision library openCV.

Test dataset

A set of 100 images that were not used for training the Bayesian model was used for evaluating the proposed algorithm. These images had a high variability in the illumination conditions, growth stages, type of crops and weed infestation rates. These 100 images were also manually segmented to evaluate the effectiveness of the proposed algorithm in segmentation.

Comparison of the proposed algorithm against the vegetation index-based methods

As one of the main objectives of this study was to compare the performance of the proposed algorithm against the state-of-the-art vegetation index-based methods, ExG (Woebbecke, 1995), ExGExR (Camargo Neto, 2004) and CIVE (Katoka et al, 2003) were chosen considering their effectiveness in vegetation segmentation. The ExG, ExGExR and CIVE were calculated using Eqs. 5, 6 and 7 respectively.

$$
\begin{gathered}
E x G=2 * G-R-B \\
\text { ExGExR }=\operatorname{ExG}-(1.4 * R-G) \\
\text { CIVE }=0.441 R-0.811 G+0.385 B+18.78745
\end{gathered}
$$

where $\mathrm{R}, \mathrm{G}$ and $\mathrm{B}$ were the normalized red, green and blue channel values of a pixel. The RGB pixel values were normalized by following a normalization scheme that usually appears in agronomic image segmentation (Woebbecke, 1995). As the images resulting from these vegetation index-based methods were grey level, Otsu's thresholding method (Ostu, 1975) was adopted to binarize the index tonal images. Some segmentation results with different algorithms are shown in Figure 2 and 3. The average accuracies of segmentation of the algorithms are displayed in Table 2. 


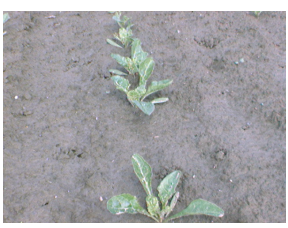

(a)

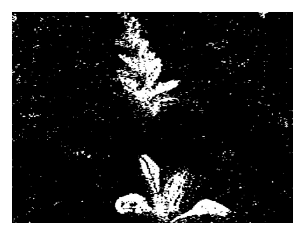

(b)

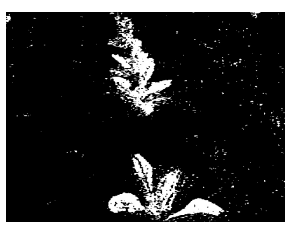

(c)

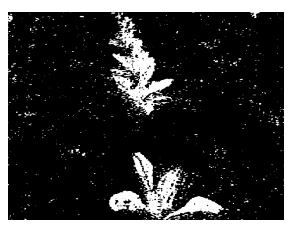

(d)

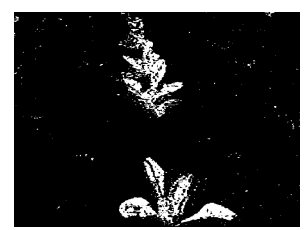

(e)

Figure 2. Segmentation results of an image taken on a cloudy day with different algorithms [(a) original image, (b) result with ExG, (c) result with ExGExR, (d) result with CIVE, (e) result with the proposed algorithm].

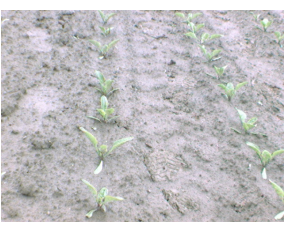

(a)

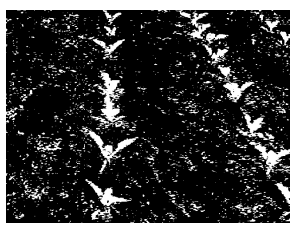

(b)

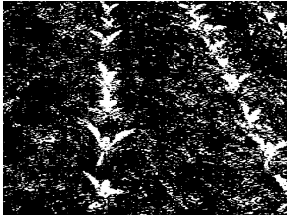

(c)

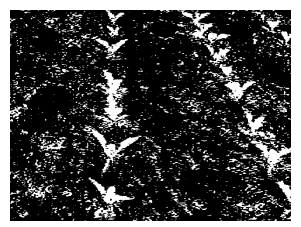

(d)

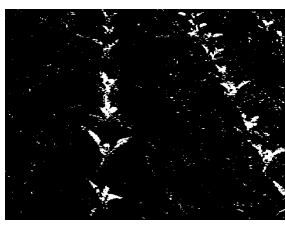

(e)

Figure 3. Segmentation results of an image with whitish background with different algorithms [(a) original image, (b) result with ExG, (c) result with ExGExR, (d) result with CIVE, (e) result with the proposed algorithm].

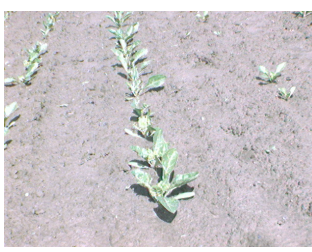

(a)

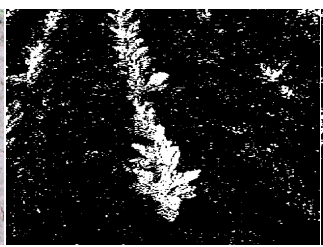

(b)

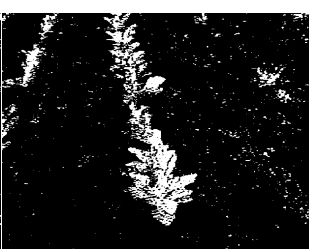

(c)

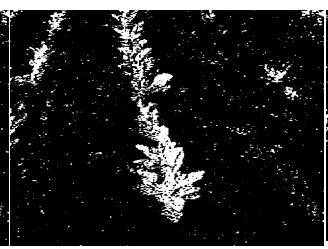

(d)

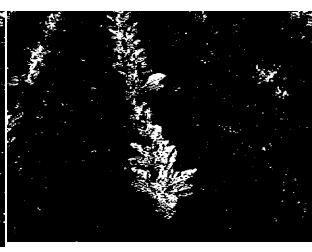

(e)

Figure 3. Segmentation results of an image taken on a sunny day with different algorithms [(a) original image, (b) result with ExG, (c) result with ExGExR, (d) result with CIVE, (e) result with the proposed algorithm].

The proposed algorithm showed no significant improvement in performance over the other vegetation index-based methods for images taken with diffused light on overcast days as shown in Figure 2. But for images with whitish background, as in Figure 3 and for images taken on sunny days, as in Figure 4, the segmentation results of the proposed algorithm were superior to the results of the three vegetation index-based methods, as vegetation index-based methods misclassified majority of the background pixels (including those of stones) as belonging to plants.

By comparing the segmentation accuracies of different methods from Table 2, it was concluded that the proposed algorithm had on average better segmentation results than the other vegetation index-based methods.

Processing time and memory usage reduction

As the resource-constrained small field robots depend on the real-time segmentation of green vegetation for various applications, the processing time and memory requirements of the segmentation algorithm is critical.

The processing time per image of the mean-shift based segmentation algorithm is about 24 
times that of the ExG- and CIVE-based algorithm making it unsuitable for real-time applications (Zheng et al, 2009). The processing time of the proposed algorithm was slightly greater yet comparable to that of the vegetation index-based methods, as shown in Table 3, making it suitable for real-time applications.

Table 2. Accuracies of different segmentation algorithms

\begin{tabular}{lllll}
\hline Algorithm & $\begin{array}{l}\text { Proposed } \\
\text { algorithm }\end{array}$ & ExG & ExGExR & CIVE \\
\hline Accuracy & 0.87 & 0.78 & 0.76 & 0.81 \\
\hline
\end{tabular}

Table 3. Processing times of different segmentation algorithms

\begin{tabular}{lllll}
\hline Algorithm & $\begin{array}{l}\text { Proposed } \\
\text { algorithm }\end{array}$ & ExG & ExGExR & CIVE \\
\hline $\begin{array}{l}\text { Processing time } \\
\text { per image }\end{array}$ & 0.54 & 0.40 & 0.41 & 0.41 \\
\hline
\end{tabular}

The proposed algorithm required only the naïve Bayesian model ( 1060 Bytes) to be stored in the memory whereas, the EASA-based algorithms store the entire look-up table (LUT), containing all the possible combination of features from the images, in the memory (Tian \& Slaughter, 1998). Therefore the proposed algorithm had both reduced processing time and memory usage compared to the other state-of-the-art segmentation algorithms without compromising on the performance.

\section{Conclusion}

In this paper, a novel Bayesian model-based segmentation of green vegetation was proposed, implemented and evaluated on real field images of sugar beet and maize plants. The naïve Bayesian classifier was trained on the manually extracted features from RGB and HSV color spaces on a set of 500 images. The segmentation accuracy was compared against the vegetation index-based methods ExG, ExGExR and CIVE. It was seen that the proposed algorithm on average performed better than the state-of-the-art vegetation indexbased methods with comparable processing time per image. Compared to the EASA-based algorithms, the proposed algorithm had reduced memory usage, making it suitable for resource-constrained real-time applications.

\section{Acknowledgements}

This research project was funded by the research platform AgricultureIsLife of Gembloux Agro-Bio Tech, University of Liège, Belgium. 


\section{References}

Camargo Neto, J. 2004. A combined statistical-soft computing approach for classification and mapping weed species in minimum-tillage systems. Unpublished Ph.D. Dissertation. University of Nebraska, Lincoln, NE, $117 \mathrm{pp}$.

Guijarro, M., Pajares, G., Riomoros, I., Herrera, P. J., Burgos-Artizzu, X. P., \& Ribeiro, A. (2011). Automatic segmentation of relevant textures in agricultural images. Computers and Electronics in Agriculture, 75(1), 75-83.

Hague, T., Tillett, N. D., \& Wheeler, H. 2006. Automated crop and weed monitoring in widely spaced cereals. Precision Agriculture, 7(1), 21-32.

Hall, M. A., \& Smith, L. A. 1999. Feature Selection for Machine Learning: Comparing a Correlation-Based Filter Approach to the Wrapper. In: Proceedings of the Twelfth International Florida Artificial Intelligence Research Society Conference (pp. 235239). AAAI Press, Orlando, FL.

Hand, D. J., \& Yu, K. 2001. Idiot's Bayes - not so stupid after all? International statistical review, 69(3), 385-398.

Kataoka, T., Kaneko, T., Okamoto, H., \& Hata, S. 2003. Crop growth estimation system using machine vision. In: Proceedings of 2003 IEEE/ASME International Conference on Advanced Intelligent Mechatronics (Vol. 2, pp. b1079-b1083). IEEE.

Meyer, G. E., \& Camargo Neto, J. 2008. Verification of color vegetation indices for automated crop imaging applications. Computers and Electronics in Agriculture, 63(2), 282-293.

Meyer, G. E., Camargo Neto, J., Jones, D. D., \& Hindman, T. W. 2004. Intensified fuzzy clusters for classifying plant, soil, and residue regions of interest from color images. Computers and Electronics in Agriculture, 42(3), 161-180.

Otsu, N. (1975). A threshold selection method from gray-level histograms. Automatica, 11(285-296), 23-27.

Rovira-Más, F., Zhang, Q., Reid, J. F., \& Will, J. D. 2005. Hough-transform-based vision algorithm for crop row detection of an automated agricultural vehicle. Proceedings of the Institution of Mechanical Engineers, Part D: Journal of Automobile Engineering, 219(8), 999-1010.

Tian, L. F., \& Slaughter, D. C. 1998. Environmentally adaptive segmentation algorithm for outdoor image segmentation. Computers and Electronics in Agriculture, 21(3), 153168.

Woebbecke, D. M., Meyer, G. E., Von Bargen, K., \& Mortensen, D. A. 1995. Color indices for weed identification under various soil, residue, and lighting conditions. Transactions of the ASAE, 38(1), 259-269.

Zheng, L., Zhang, J., \& Wang, Q. 2009. Mean-shift-based color segmentation of images containing green vegetation. Computers and Electronics in Agriculture, 65(1), 93-98. 